\title{
Potential of natural astaxanthin in alleviating the risk of cytokine storm and improve health in COVID- 19: A scoping review
}

Jayanta Talukdar ( $\sim$ jayanta.talukdar@ril.com )

Reliance Research \& Development Center, RIL

Bhaskar Bhadra

Reliance Research \& Development Center, RIL

Santanu Dasgupta

Reliance Research Development Center, RIL

Vinod Nagle

Reliance Research Development Center, RIL

\section{Systematic Review}

Keywords: COVID-19, Cytokine Storm, Acute Respiratory Distress Syndrome, Astaxanthin, Anti-oxidant, Anti-inflammatory

Posted Date: May 5th, 2020

DOl: https://doi.org/10.21203/rs.3.rs-26458/v1

License: (9) This work is licensed under a Creative Commons Attribution 4.0 International License. Read Full License 


\section{Abstract}

Background: Natural astaxanthin as a potent anti-oxidant and broad-spectrum anti-inflammatory bioactive molecule plays important role in modulating the immune response, speculated to be a potential supplement to alleviate cytokine release storm in COVID-19.

Objective: Review of published literature to summarize the rationale for possible benefits of natural astaxanthin to support COVID-19 patients.

Methods: Retrieved relevant literature from electronic databases including Google scholar, PubMed, Scopus, etc. and reviewed following Preferred Reporting Items for Systematic Reviews and Meta-Analysis (PRISMA) Statement. We adapted the article as scoping review.

Results: Cytokine release syndrome (CRS) is reported as a common feature in COVID-19, which can lead to potentially fatal, hyper-inflammatory acute respiratory distress condition, diagnosed with elevated serum level of pro-inflammatory cytokines like IL-6, CRP, etc. that positively correlated with disease severity. Anti-inflammatory drug, like tocilizumab, etc. are under clinical trials as anti-CRS therapy. Astaxanthin can potentially alleviate CRS by regulating inflammatory cytokines by inhibiting the activities of NF-kB, TNF-a, JAK/STAT-3, etc. Available pre-clinical and clinical trials data support its excellent safety, and potential therapeutic and health benefits.

Conclusions: Natural astaxanthin has tremendous potential as co-adjunctive supplement, desiring necessary clinical supports on its efficacy and beneficial against COVID-19.

\section{Introduction}

The recent pandemic outbreak of Severe Acute Respiratory Syndrome Coronavirus-2 (SARS-CoV-2) induced coronavirus disease-2019 (COVID-19) has imposed tremendous challenge to mankind. Without any definitive treatment till date, the disease has spread rapidly over 200 countries, infected more than 2.5 million people and claimed over 200 thousand lives globally (1). Although the pathophysiology of SARS-CoV-2 is yet to be studied well, available evidences suggest similar features to SARS-CoV and MERS-CoV; SARS-CoV-2 infection triggers local or systemic inflammation leading to aggressive inflammation or the cytokine release syndrome (CRS). In severe progression, the disease results in radiographic evidence of pneumonia, with acute lung injury (ALI) or acute respiratory distress syndrome (ARDS), respiratory failure, sudden cardiac arrest, sepsis, etc. (2-7).

Natural astaxanthin (3,3'-two hydroxyl-4,4'-two ketone-beta, beta'-carotene) (Fig.1), a non-provitamin A keto-carotenoid of predominantly marine origin, is a potent antioxidant and anti-inflammatory molecule as demonstrated in both experimental and human studies (8-12). Unlike the chemically synthesized disodium di-succinate astaxanthin (DDA), commonly known as synthetic astaxanthin, microalgae derived natural astaxanthin (nAXN) was reported superior with stronger antioxidant activity, higher bioavailability, extensively studied, proven safe for human consumption and used in a wide array of health- 
promoting clinical, nutritional and functional food supplements (8-10). nAXN is an esterified form with natural fatty acid(s), which makes it potent antioxidant and highly bioavailable (8-10). Clinically, nASX shown diverse benefits with excellent safety. Previous studies reported its abilities to block oxidative DNA damage, lowered CRP and other inflammation biomarkers including IL6 and TNF- $a$ activations, increase immune response by increasing $T$ and $B$ cells subpopulations and inhibit NF-kB signaling pathway (8-9, 11-15). Studies in animal model also demonstrated that $n A S X$ exerts protective effects against LPSinduced sepsis, ARDS, ALI via inhibiting the expression of pro-inflammatory cytokines through MAPK/NF$\mathrm{kB}$ signaling pathway (12). The intervention of NF-kB mediated inflammatory response was suggested as one of the potential approaches to treat and prevent septic shock, and related complications (12). Further evidences on the abilities of nASX to downregulate the expression of pro- inflammatory cytokines, IL-6, IL$\beta$, COX2, CRP, and TNF- $a$ suggest its potential role in the treatment and alleviating CRS-induced inflammatory disease such as ALI/ARDS and sepsis caused due to bacterial and viral infection (12-13).

Although there are available literature suggesting nASX may be beneficial in the treatment of ROS induced oxidative stress, acute lung injury, sepsis and cytokine storm caused by avian influenza virus infection (13), no reports are available on its possible application against corona virus or COVID-19. Herein, we aimed to review and summarize the relevant evidences on therapeutic benefits of nASX emphasizing about pathogenic or non-pathogenic cytokine storm and its related diseases suggesting nASX may be of therapeutic value in COVID-19.

\section{Methods}

\section{Study design, search strategy and selection criteria}

Relevant information on therapeutic and health benefits of astaxanthin, pathogenesis and clinical presentation of COVID-19 were retrieved from Google scholar, PubMed, Scopus, etc. till April 23, 2020. A scoping review of the relevant literature was conducted to summarize the rationale for anticipating nASX as a potential co-adjunctive supplement for COVID-19 based on understanding of its putative pathogenesis and clinical presentations in symptomatic cases following the guidelines stated by Arksey and O'Malley (16). The rationale for potential therapeutic and other health benefits of nASX relevant to COVID-19 were reviewed and reported based on available relevant scientific information following the guidelines of PRISMA (17). No ethical approval was obtained as all relevant information were retrieved from publicly available literature through multiple databases and no primary data was collected or generated during the review process. The studies included with relevant literature describing the clinical characteristics, epidemiology, prevention or control or treatment of COVID-19. Literature on astaxanthin with relevant information were also retrieved. Here we use specific search phrase like "astaxanthin ARDS", "astaxanthin ALI", "astaxanthin antivirals", "astaxanthin anti-inflammatory", etc. We included all relevant scientific publications written in English for this review.

\section{Screening, data extraction and summarizing the findings}


Eventually after screening and removing duplication, 86 articles were selected for the intended review. Selected articles were categorically reviewed and extracted data were recorded, broadly under the domains: epidemiology, clinical manifestation and diagnosis, and prevention, control and treatment for COVID-19. In case of articles related to nASX were categorized broadly: anti-COVID-19, anti-inflammatory, anti-oxidant, immunomodulator and protection against CRS, ARDS, ALI.

Based on the principal research objective of the study, articles were classified as mentioned above. Information based on experimental findings and conclusions as stated in the relevant articles supporting as evidence were reported in the review result. All findings and statements regarding potential use of nASX as adjunctive supplement in COVID-19 mentioned herein this review are purely based on published literature as listed in the references. There is no available study related to nASX against coronavirus or SARS-CoV-2 or COVID-19, which we consider as the major limitation of this study. However, presence of multiple pre-clinical and human studies reporting indirect evidences supporting possible therapeutic implications of nASX as anticipated, are the major strength of this review.

\section{Results}

The search result revealed that pro-inflammatory cytokine storm is a common feature among severe COVID-19 infection. The reports suggested that CRS mediated hyperinflammatory ARDS, Sepsis, ALI and multiorgan failure are the major cause of death. We included total 86 reports, which were categorized into the selected research domains (S1, Supplementary file). $40.6 \%$ of the total articles were related to COVID19. About $25.5 \%$ of the reports were related to the epidemiology, clinical and pathological features including diagnosis of COVID-19. 15.1\% of the reports mentioned about possible applications of Chinese Traditional Medicines (CTM) and other natural compound in combination therapy and repurposing antivirals and anti-inflammatory drugs for possible treatment, prevention or control. None of the search result reported any studies directly related to the effectiveness of nASX in treatment of COVID-19, which is understandable as the outbreak is recent our understanding in it is still evolving. However, $59.4 \%$ of the total retrieved articles stated potential therapeutic and health benefits of nASX as anti- inflammatory, antioxidant and immunomodulator, which altogether exerts protective activities against cytokine storm and associated diseases.

\subsection{Clinical features of SARS-CoV-2 infections}

Huang et al. (3) reported that SARS-CoV-2 infected symptomatic patients revealed with upper respiratory infection symptoms included, fever, fatigue, nonproductive cough, myalgia, as common symptoms of COVID-19. Although the complex pathological mechanism involved in COVID-19 that produces pneumonia is evolving rapidly, accumulating evidences from early reports on clinical and laboratory features from severe COVID-19 patients revealed the presence of a CRS, resulting in ARDS, sepsis and multi- organ failures $(2-3,5-6)$. CRS is the deadly uncontrolled systemic inflammatory response resulting from the release of large amounts of pro-inflammatory cytokines including IL-1 $\beta, I L-6$, TNF-a, TGF $\beta$, IL-10, 
IFN- $\gamma$, etc., and chemokines such as CCL2, CCL3, CCL5, etc. (2-7, 18-23). Available reports suggested that elevated serum concentration of cytokine IL- 6 and other inflammatory cytokines are hallmark of CRS, which is common in COVID- 19 infection $(2-3,6)$. Elevated level of serum IL- 6 and CRP that correlate with respiratory failure, ARDS and other clinical adversities, are the biomarkers of severe COVID-19 infection $(2-3,6)$. Liu et al. (2) reported increased cytokine levels in 69 severe COVID-19 inpatients of median age of 56 years, elevated level of CRP, IL-6, LDH and ferritin were more prominent and significantly corelated to severe immune damage to the lung and multiple other organs. Mild variation in the serum level of IL-2, IL4, IL-10, IFN-y, and TNF-a were reported among severe and non-severe patients (2). Intriguingly, based on the comparison of IL-6 level in 30 patients before and after treatment, Liu et al. (2) reported that the IL-6 level was significantly reduced with disease improvement as revealed by CT assessment, while persistent increase of IL- 6 was recorded with disease progression. With the remission of disease, the IL- 6 level along with D-dimer, CRP and LDH levels were also reported to decrease significantly (2). These observations clearly suggest that lymphocytopenia and CRS are associated with the severity of COVID19.

In another study, Liu et al. (23) reported that hypoalbuminemia, lymphopenia, decreased percentage of lymphocytes and neutrophils, elevated CRP and LDH, and decreased CD8+ T cell count were common abnormalities among the severe COVID-19 patients developed ARDS.

The CRS triggers a violent attack by the immune system to the body, causing acute lung injury (ALI), RNAaemia, acute cardiac injury, sepsis and multiple organ failure, and finally lead to death in severe cases of SARS-CoV-2 infection (2-3, 5-6, 10, 18-24). The CRS results because of an accentuated immune response triggered by SARS-CoV-2 infection in severe COVID-19 patients, diagnosed with lymphocytopenia, a differential diagnostic criterion for COVID-19 (2-3, 5-6, 22). The CRS caused by SARSCoV-2 was reported to mediated by leukocytes other than T cells, which may be overcome by blocking IL$1, \mathrm{IL}-6$ and TNFa $(2,6,22-23)$. Hence, destroying the immune evasion of SARS-CoV-2 is imperative in its treatment and specific drug development $(6,22)$. Based on understanding of COVID-19 triggered CRS, it is pivotal to treat the infection (with appropriate anti-viral therapy), and it may be even more critical to treat the host with appropriate therapeutic modalities to dampen the overly exuberant immune responses responsible for CRS-induced multi-organ dysfunction syndrome (MODS) and death (2-3, 5-6, 22-27). Zhou et al. (27) from a retrospective cohort study of 119 hospitalized patients reported that older age with comorbidities and elevated level of d-dimer were at higher risk of COVID-19 fatality. Besides elevated level of IL-6, high-sensitivity cardiac troponin- I, LDH, and lymphopenia in severe COVID-19 illness, occurrences of sepsis was reported to be common complications (27).

\subsection{Current knowledge of antiviral and anti-inflammatory treatment for COVID-19}

Currently, there are no clear expert consensus or guidelines on how to treat SARS-CoV- 2 induced COVID19. Antiviral and supportive management with specific respiratory and ventilator supports are current 
mainstream of treatments for severe cases. Repurposing antiviral drug along with corticosteroid therapy are considered for risk-adapted treatment for severe COVID-19 patients. With the lack of a definitive treatment, sequential progression of respiratory compromise was the predominance of respiratory malfunction in overall clinical demise $(2,19-21,24-27)$. As CRS is relatively a common manifestation in COVID-19 illness and often lead to exacerbation with progression to ARDS, intervention with antiinflammation therapy in addition is pivotal importance to prevent further injury $(2-3,5-6,22,26-27,29-30)$. Shi et al. (22) suggested a two-phase approach for potential treatments of COVID-19 patients: the first immune defense-based protective phase for non-severe COVID-19 cases and the second, inflammationdriven damaging phase for severe COVID-19 patients. Given the viral nature of CRS in COVID-19, prompt initiation of the anti-inflammatory treatment at an early stage based on the clinical and immunological characteristics of a severe patient can contain abrupt deterioration (35).

Present treatment approaches for COVID-19 are based on treatments for SARS- CoV infection and the ensuing chronic inflammation include anti-viral medications as well as steroidal and non-steroidal antiinflammatories (NSAIDS), glucocorticoids', hydroxychloroquine or chloroquine, TNF-inhibitors and antibiotics. $(2-3,6,26-35)$. Alternative treatment with antiviral Chinese Traditional Medicine (CTM) were also reported under clinical trials $(26-27,29)$. Zhang et al. (36) proposed melatonin as an adjuvant for COVID-19 based on its known anti-inflammatory and anti-oxidant properties. Of note, the prognosis and recovery from critical hyperinflammation stage of illness is very poor, and prompt recognition and application of preventive therapy may yield better results $(6,26-27,34-35)$. We anticipated that a range of natural anti-inflammatory and antioxidants as supplements will offer a window of quick recovery of patients by reducing post treatment side-effects.

While the anti-viral approaches and vaccines being considered as immediate countermeasures are unavailable and undergoing intense researches, there may be needed to consider treatment regimens from analogous disease patterns $(6,22,26,29-31,34-36)$. Matching clinical dispositions may be considered in efforts to develop therapeutic interventions. Additionally, dire outcomes of illness may be overcome with adjunctive measures that do not necessarily cure the underlying disease $(6,22,26-31)$. Repurposing an old malaria drug chloroquine or hydroxychloroquine was reported to show potent activity against COVID-19 and reported to use as preventive medicine to treat COVID-19 patients and medical professionals $(25,27-28,30-31)$. More than a supportive care, an adjunctive measure may assist severe COVID-19 patients health (31). Based on supportive evidences there are multiple natural compound which may have advantages to use as adjunctive countermeasures for rapid recovery of COVID-19 patients (6, 26). Herein, we outline the strong evidences of natural astaxanthin as a potent anti-inflammatory and anti-oxidant compound supporting its potential beneficial application as adjunctive in patients with COVID-19.

\subsection{Rationale for use of astaxanthin as potential adjunctive supplement}


Microalgae Haematococcus pluvialis derived natural astaxanthin (nASX) is a unique bioactive molecule with diverse clinical and health benefits (10). Previous studies reported that nASX, which the microalga $H$. pluvialis accumulated in response to adverse conditions, can play major roles in regulating immunity and disease etiology, suggesting its wide array of potential therapeutic and nutritional supports as antioxidant, immune- booster, anti-inflammatory, neuroprotective, immunomodulatory, anti-proliferative, anti-aging, anti-bacterial, anti-apoptotic, etc., (8-11, 14-15, 37-45). Figure 2, represents an outline of H.pluvialis biomass generation, accumulation of astaxanthin and recovery.

The pathogenesis of SARS-COV-2 is likely involve four major steps: The renin- angiotensin (RAS) signaling pathway, oxidative stress and cell death, cytokine release storm and endothelial dysfunction (46). Based on available published information, Table 1 lists some of the possible roles of nASX for the clinical characteristics of diseases associated with the increased risk of COVID-19 fatalities and how functionally $\mathrm{nASX}$ is related to some of them. As there are no direct evidences of applying nASX against COVID-19, herein, we summarize (Table 2) the most likely benefits for nASX supplementation in reducing the clinical effects of COVID-19 infection found from available studies related to the treatment of other diseases.

\section{Discussion}

\subsection{Astaxanthin protects against ROS and Oxidative Stress}

Reactive oxygen species (ROS) play a crucial role in the host inflammatory response and cytokine outbreak during various diseases including virus infections (12-14). ROS- mediated oxidative stress is correlated with the viral infection that modulates the intracellular redox sensitive signaling pathways to promote viral replication and pathogenesis, suggesting anti-oxidant as an important medicine to ROSrelated diseases (12-14, 37-38).

The pathogenesis of SARS-COV-2 begins with the dysregulation of the renin- angiotensin system (RAS) signaling pathway. RAS acts as a double edge sword, as a receptor for the virus entry and a negative regulator for severe symptoms of infection and lung injury (46). Liu et al. (23) reported abnormal increase of Angiotensin II (ANG-II) in COVID-19 severe patients which strongly corelated with multiple organ failure. It is well documented that increased ROS and resulting oxidative stress in ANG-II associated diseases results from NADPH oxidases, mitochondrial dysfunction and inflammation, including reduction of endogenous antioxidants $(37,42)$, which commonly occurs as ANG-Il pathological conditions, such as hypertension, atherosclerosis, and diabetes (47). Redox-sensitive transcription factors, such as NF-kB and activator protein-1 were reported to induce the expression of pro-inflammatory cytokines associated with ANG-II related disease, such as cardiovascular disease (48). Furthermore, ANG-II induced ROS stimulate matrix metalloproteinase (MMPs) that regulate the extracellular matrix and vascular remodeling (49). Under normal conditions, the ROS generation is balanced by the rate of elimination through endogenous and dietary antioxidants, which greatly imbalanced by elevated ROS generation with the dysregulation of ANG-II signaling and contributes to cardiovascular dysfunction including heart failure, stroke, 
hypertension, etc. $(42,49)$. This may also relate the potential COVID-19 high case fatality rate for patients with chronic comorbidities like hypertension $(6 \%)$ and cardiovascular disease $(10.5 \%)(3,23,50)$.

Oxidative stress is an important molecular mechanism underlying pulmonary fibrosis. Accumulated evidences suggest that ROS released by activated phagocytes causes lung inflammatory process, leading to lung injury (51-52). Song et al. (52) demonstrated the anti-oxidative and anti-fibrotic effect of nASX in mouse model in vivo and in vitro and reported that nASX blocks ROS generation and dosedependent apoptosis in alveolar epithelial cells type II, as characterized by inhibition of cytochrome- $\mathrm{C}$ (Cyt C) release and the activation caspase-9, caspase-3, Nrf-2 and other cytoprotective genes. With its unique molecular structure nASX stretches through the bilayer membrane, provides resilient protection against oxidative stress (9). Unlike most antioxidants, which work either in the inner (e.g., vitamin E and $\beta$ carotene) or the outer side of the membrane (e.g., vitamin C), nASX can scavenge and quench ROS and free radicals (superoxide anion, hydrogen peroxide, singlet oxygen, etc.) in both the inner and outer layers of the cellular membrane $(9-10,38)$.

\subsection{Astaxanthin protects against inflammation}

The anti-oxidative effect of nASX cooperates with its anti-inflammatory actions by up- regulating antioxidative enzymes (e.g. superoxide dismutase) and down-regulating pro- oxidative enzymes (e.g. nitric oxide synthetase) $(8,37,47-53)$. Astaxanthin is also known to protect pancreatic beta cells by reducing oxidative stress and sugar toxicity, improve the levels of adiponectin and HDL, and enhances blood flow and circulations (14). nASX exerts broad-spectrum anti-inflammatory activities through multiple pathways $(9,14,40,46-57)$. nASX significantly attenuate pathological elevation, critical inflammatory cell signaling nuclear factor kappa-B (NF-kB) pathway both in vitro and in vivo and reduce TNF-a in humans, resulting decrease in multiple pro-inflammatory cytokine level, which may potential in maintaining lung health and minimizing the impacts of SARS-CoV-2 infection (12-14). It was well documented from studies in animal models that $n A S X$ shows significant decrease of important mediators of inflammation including, IL-6, IL-1b, COX-2, CRP, PGE-2, iNOS, and nitric oxide (NO) (12-14, 37-40, 42-46).

Miyachi et al. (9) reported that following nASX treatment, localization of NF-KB/p65 and the level IL6 and TNF-a decreased, while significant improvement of cell proliferation in vitro. Oral lichen planus (OLP) is a chronic inflammatory disease associated with viral infection such as hepatitis $\mathrm{C}$ and human immune deficiency virus (HIV) (9). The pathogenesis of OLP involves, a T-cell mediated autoimmune reaction in which cytotoxic CD8+ T cells trigger apoptosis of keratinocytes and cytokines IL-1, IL-2, IL-6, IL-8 and TNF- $a$ contribute in upregulation of adhesion molecules $(9,58-60)$. Based on these observations, we hypothesized that curative administration of nASX in SARS-CoV-2 induced lung infection may suppress the vicious cycle of inflammatory stimulus by protecting the bio-membrane, resulting an increase in cell proliferation in later stage.

Recently, Yang et al. (61) reported the anti-inflammatory effects of various astaxanthin isomers and stated that nASX achieved anti-inflammatory activity by modulating the NF-kB signaling pathway to 
down-regulate TNF-a induced phosphorylation of IkB-a. Chan et al. (62) demonstrated that diet containing $0.05 \% \mathrm{nASX}$ supplied to diabetic rat retained glutathione content in plasma, significantly decreased plasma levels of CRP, reduced ROS, IL- 6 and TNF-a, MCP- 1 and significantly diminished plasminogen activator inhibitor-1. These results supported that nASX can potentially attenuate coagulatory, oxidative and inflammatory stress, which may show similar beneficial effects in COVID-19 patients comorbid with diabetic conditions.

Acute inflammatory disease such as sepsis and acute lung injury (ALI) is a systemic inflammatory syndrome induced by infection and involves damage to multiple organs and tissues (12). ALI is one of the major causes of SARS-CoV-2 induced COVID- 19 fatalities $(2-6,18-23,26-27)$. ARDS is the acute condition of a COVID-19 infected patients leading to sudden breathlessness, rapid heart rate, severe hypoxia, and requiring immediate intensive care facilities. COVID-19 patients suffering from ARDS end up with damaged alveolar walls called diffuse alveolar damage leading to serious scarcity of oxygen supply to the other organs through the blood resulting potential multiple organ failures $(2-3,6,21)$. Research efforts currently have been largely focused on innate immune system and conceptually viewed sepsis and ALI as syndrome of hyperinflammation (2, 5-6, 18-21). Evidences from lipopolysaccharide (LPS)induced acute lung injury and sepsis (12), suggest the activation of pro-inflammatory signaling pathways, including transcription factor nuclear factor kappa-light chain-enhancer of activated B cells (NF-KB) and mitogen activated protein kinases (MAPKs), triggering the production of a variety of inflammatory cytokines (12-13). The excessive release of various pro-inflammatory cytokines mainly including TNF-a, IL-6, IL-1 $\beta$, IL-12 and IL-8 rapidly initiate a systemic inflammatory response leading to simulation of adaptive immune response and cytokine storm resulting the acute cellular injury to form sepsis or ALI $(6,12-13)$. Applications of anti-inflammatory / anti-oxidants candidates to intervene the excessive production of cytokines, including IL6 and TNF-a can be a promising strategy in prevention and treatment of COVID-19 induced ARDS related ALI and sepsis $(2-6,26,35)$. With its proven antiinflammatory and antioxidant activities nASX may be considered as a promising candidate to be trial against COVID-19. Previous studies reported the potential applications of nASX in the treatment of avian influenza virus infection and stated that $\mathrm{nASX}$ can exhibit significant benefit in a combinatorial drug approach along with antiviral compound to be used for the treatment (12-13). Although, detail clinical result is lacking at this moment, pre-clinical trials in animal model was demonstrated with encouraging results. Cai et al. (12) demonstrated that nASX can prevent LPS-induced ALI/ARDS and sepsis by inhibiting the activation of pro- inflammatory signaling pathway of MPAK/NF-KB. Experimental results demonstrated that nASX significantly inhibits the production IL6 and TNF-a. The results showed that treatment with nASX not only significantly reduced the death rate due to sepsis but also exerted protective effects on lung tissues. nASX treatment also decreased alveolar wall swelling and lessened the decline in the number of pulmonary alveoli in lung tissue (12).

With the absence of definitive treatment for acute COVID-19 infection, ARDS/ALI are leading with high case fatality rate $(2-3,5-6,27,34)$. Sepsis syndrome is the most frequent causes of ARDS, leading to increased lung permeability, enhanced polymorphonuclear neutrophil (PMN) sequestration and respiratory failure causing sudden rise in death toll, as indicated by current pandemic worldwide $(21,35$, 
63-65). During acute COVID-19 treatment in intensive care unit, high dose of vitamin $\mathrm{C}$ was suggested as a "rescue therapy", along with high pressure flow nasal oxygen (63). Administration of anti-inflammatory substance to potentially avert the existing ARDS condition is not known (35). Taken together, we speculate that use of nASX as adjunctive supplement in the treatment of COVID-19 may exert dual purpose of both as anti-oxidant and anti-inflammatory compound with beneficial outcome of reduce fatality and rapid recovery.

\subsection{Astaxanthin as immunomodulatory and immune booster}

Cytokine storms, which rapidly cause life-threatening single or multiple organ failure are, considered to be one the notable causes of death for severe COVID-19. SARS-CoV-2 infection rapidly activate pathogenic T cells and produce granulocyte-macrophage colony stimulating factor (GM-CSF) and IL-6. GM-CSF further activate CD14+ and CD16+ inflammatory monocyte and elevate IL- 6 production and other inflammatory factors resulting in a cytokine storm marked by very high level of inflammatory markers for critically ill COVID-19 patients $(2,35)$. Clinical characteristics of COVID-19 suggest a reduced level of neutrophils, lymphocytes, CD4+ T and CD8+ T cells in peripheral blood along with very high level of inflammatory factors indicates disease severity leading to ALI, ARDS and potential death $(2-6,35,66-67)$. Zhang et al. (35) reported that severe COVID-19 patients diagnosed with very high level of erythematosus sedimentation (ESR), CRP, IL-6, TNF- $a$, IL-1 $\beta$, IL-8, etc., associated with ARDS, hypercoagulation and disseminated travascular coagulation (DIC), manifested as thrombosis, etc. At critical stage the markers of systemic inflammation reported to be extremely elevated, which suggest the importance to block CRS at the right window of time to initiate anti- inflammatory therapy to contain COVID-19 death rate (35).

The immune boosting activities of nASX have been well documented and supported by pre-clinical and clinical trials including human models $(9,14,40,45,68-70)$. As a potent antioxidant and antiinflammatory molecule with known immunomodulatory activities nASX may plays a pivotal role in modulating the immune response in COVID-19. Park et al. (14) reported that dietary supplement of nASX stimulate mitogen-induced lymphocyte proliferation, increase natural killer cell cytotoxicity and the delayed-type hypersensitivity response, and increase the number of total $\mathrm{T}$ and $\mathrm{B}$ cells in the peripheral blood. It was also reported that astaxanthin is absorbed after oral administration subsequently utilized by blood leukocyte subcellular organelles, mostly by the mitochondria $(14,68)$. Notably, dramatic decreased of DNA damage biomarker (plasma 8-OHdG), along with significant reduction of plasma C-reactive protein concentrations were reported (14). Lin et al. (70) reported that nASX modulates the production of T helper 1 cytokines, such as IL-2 and IFN-y, without causing significant cytotoxic effects in primary cultured lymphocytes. Reports also suggest that nASX exerts regulatory actions on the immune system and directly enhances the immune response by improving proliferation and maturation of natural killing cells, $T$ and B lymphocytes, granulocytes and monocytes $(14,68-70)$. Diao et al. (71) in mice model demonstrated that administration of nASX significantly induced rapid accumulation CD8+ T cells 
accompanied by elevated expression of IFN-y and alleviated renal fibrosis. It was reported that nASX increase CD8+ $T$ cells population by upregulating the expression of CCL5 in macrophages (71).

Immunomodulation by natural bioactive compound can provide additional therapeutic support to conventional chemotherapy for a range of diseases including COVID-19, especially when selective immunosuppression is needed for autoimmune disorders. There are several diseases where immunostimulatory drugs are needed to overcome the immunosuppression induced by drugs or environmental factors, and immunosuppressants are required when there is undesired immunopotentiation. Moreover, drugs that can improve the immune system are needed to quell the immunosuppressive effects produced by stress and chronic diseases, and in situations where immune responsiveness is impaired such as COVID-19 (72).

\subsection{Astaxanthin as potential therapeutic supplement against COVID-19}

Accumulated evidences suggest CRS as the major cause of morbidity in SARS-CoV-2 infected COVID-19 disease, which urgently needed therapies based on suppressing CRS (6). Current management of COVID19 is supportive, and respiratory failure from ARDS is the leading cause of death. Elevated level of serum IL-6, TNF-a, CRP, etc. were recognized as the predictors of fatality in severe COVID-19 cases. Moore and June (6) reported that proinflammatory IL-6 plays a critical role in aberrant activation of JAK/STAT3 (Janus kinase/signal transducer and activator of transcription 3), resulting a systemic cytokine storm involving secretion of vascular endothelial growth factor (VEGF), which contributes to vascular permeability and the extravasation of immune cells from blood vessels (6). Mehta et al. (73) suggested that JAK inhibition can affect both inflammation and cellular viral entry in COVID-19, which possibly can reduce the case fatality rate of severe COVID-19 due to hyperinflammatory syndrome characterized by a fulminant and fatal hypercytokinaemia with multiorgan failure. Intriguingly, in a study using human endothelial cell line ECV304 by Kowshik et al. (74) reported that dietary nASX is a potent inhibitor of JAK/STAT3. It was reported that nASX abrogated STAT3 by downregulating VEGF and VEGFR2. The study also reported that dietary nASX significantly reduced the expression of matrix metalloproteinases (MMPs), MMP2 and MMP9 (74). As discussed herein, available published information suggests the potential use of nASX in the treatment of COVID-19 with the subject related to other disease-causing systemic inflammation, ROS burst, etc., reported to exert promising results in attenuation of inflammatory cytokines levels (11-15, 42-47, 51-57, 61-62). As discussed above, Cai et al. (12) demonstrated the potential use of nASX in the treatment of clinical sepsis and reported that treatment with nASX exhibited significant protection against ALI/ ARDS, repressed alveolar wall swelling and attenuated the decline in the number of pulmonary alveolar via inhibition of pro-inflammatory cytokine such as TNF-a and IL- 6 secretion, inhibition of MAPK/NF-kB signaling pathway, suppressing the degradation of IKB- $\alpha$ and phosphorylation of ERK1/2, P38 and JNK, decreasing Myeloperoxidase (MPO) activity, reducing macrophage infiltration as evidenced by reduced CD38 expression, and inhibiting bronchial alveolar lavage fluid (BALF) (12). 
Respiratory failure from ARDS, with its poor prognosis and lack of an effective clinical therapy is leading cause of death of acute COVID-19 patients $(2-3,6,20-21,35)$. Steroidal (e.g. Corticosteroids) and nonsteroidal anti-inflammatory drugs (NSAIDs) were reported to be either therapeutically ineffective or exacerbate symptoms in the treatment of sepsis and ALI in acute COVID-19 patients $(6,35,74)$. Application of nASX can be a potential approach to intervene the inflammatory responses for the treatment and prevention of CRS and its associated ailments in COVID-19 patients.

\subsection{Astaxanthin as neuroprotective molecule}

nASX is considered as a broad-spectrum bioactive compound with its myriad of health beneficial activities exerts prudently through multiple pathways. Besides its effectiveness as controlling oxidative stress, hyperinflammation, immune response, etc., as evidenced by multiple studies, nASX is also reported as a potential neuroprotective. In this regard, and mainly due to its capability to cross blood-brain barrier, nASX has gained growing interest as a multi-target pharmacological agent in protection of CNS injury and against neurological disorders including Parkinson's disease, Alzheimer's disease, brain and spinal cord injuries, neuropathic pain, aging, depression, and autism (39-40, 45, 55, 75-76, 80-82).

Very recently Wu et al. (77) reported the neurotropic properties of SARS-CoV-2 and possible cause of neurological diseases, including viral encephalitis, infectious toxic encephalopathy, and acute cerebrovascular disease. Moreover, a growing number of COVID-19 patients were reported to loss of smell and taste (77). Mao et al. (78) reported that some patients developed COVID-19 related symptoms only after revealing neurologic symptoms. Potential threat to the damage of central nervous system caused by SARS-CoV-2 was recently confirmed by Xiang et al. (79). The pathology of severe COVID-19 infection is linked to the systemic inflammatory response syndrome (SIRS) associated with CRS (77), which suggest early anti-inflammatory intervention may effectively prevent immune damage and reduce of the risk of injury in the nervous system $(73,77)$. Effective therapeutics with the ability of multi-target treatments that simultaneously attenuate neuronal inflammation, oxidative stress and apoptosis are considered effective for treatment of neurological diseases. In this regard, nASX, based on its potent anti-oxidative, anti-inflammatory and anti-apoptotic properties, has gained tremendous interest as a multi-target pharmacological target (75). Clinically, CNS injury is characterized with primary phase involving direct death neuronal cell followed by the secondary phase, consisting of inflammatory, oxidative, apoptotic and other molecular pathways causing further damages to the neuronal cells. Zhang et al. (80) reported that nASX exerts its CNS protective role via decreasing malondialdehyde (MDA) and increasing glutathione (GSH) and SOD in rodent models. Fakhri et al. (81) reported in a rat model that nASX prevented tissue and neuronal damages by downregulating NR2B, TNF- $a$ and p-p38MAPK, and reported to improved sensory-motor function. In another study, Zhang et al. (55) reported that nASX exert anti-inflammatory effect through increasing Sirtuin 1 (SIRT1) and inhibiting the Toll-like receptor 4 (TLR 4) signaling pathway resulting reduction of pro-inflammatory response and secondary brain injury. TLR4 express mainly in microglia and plays a pivotal role in triggering inflammatory response in the CNS (55). Further, in a rat model, it was reported that nASX down- regulated matrix metallopeptidases-9 (MMP- 
9), which was attributed to the decrement in the level of, infiltering neutrophils, activated microglia, TNF-a and IL-1 $\beta$ (82).

The therapeutic implications of nASX as neuroprotective molecule has been well documented. Its possible role in treatment of CNS injury was demonstrated in animal models. Taken together, it is anticipated that $\mathrm{nASX}$ will be useful in developing a potential therapy along with specific antivirals against the potential threat neurological infection by SARS-CoV-2 and its associated disease.

\subsection{Broad spectrum health benefits of natural astaxanthin}

NOD-like receptor 3 (NLRP3) inflammasome is part of the innate immune response during lung infection (83). The pathogen, including a virus (CoVs have not yet been tested), triggers NLRP3 activation to amplify the inflammation. Emerging studies have shown ROS as the key molecule that activate NLRP3 inflammasome, which plays a pivotal role in the development of contrast-induced acute kidney injury (ClAKI) leading to renal failure, another major cause of co-morbid death in acute COVID-19 $(3,20,83,84)$. Gao et al. (83) demonstrated that nASX can attenuate iohexol-induced human proximal renal tubular epithelial cells (HK-2) injury via the mechanism related to the inhibition of ROS production and downregulation of NLRP3 inflammasome and its downstream apoptosis and inflammatory response. The experimental results confirmed that pretreatment with nASX can inhibit the overexpression of ROS in HK2 cells, thereby inhibiting the NLRP3 inflammasome and its downstream apoptosis and inflammatory response (83).

Ochratoxin (OTA), a mycotoxin produced by secondary metabolism of fungi such as Penicillium and Aspergillus, is classified as a human carcinogen in Group 2B. OTA can cause multiple organ damage including lung, kidney, liver, etc. Xu et al (85) studied the possible protective measure of nASX against OTA induced oxidative damage and inflammation in mouse model. It was reported that astaxanthin protect lung injury from OTA induced inflammation and oxidative damage via regulating Nrf2 and NF-kB pathway. Lung fibrosis is associated with inflammation characterized by the recruitment of macrophages, neutrophils and lymphocytes in the airways. nASX also reported to has therapeutic and prophylactic potential in the airway inflammatory response associated with chronic obstructive pulmonary disease (COPD) (86). Kubo et al (86) demonstrated is mice model that nASX prevent the oxidative damage via activation of Nrf2 pathway. Accumulated evidences based on published reports suggest that nASX may support with preventive measures against COVID-19 induced renal injury, septic cardiomyopathy, and liver injury.

Based on available information it is clearly understandable that astaxanthin can play a very pivotal role in regulating the CRS and may help in preventing the associated disease in multiple way (Fig 3). We presume, its potent anti-oxidant, anti-inflammatory and immunomodulatory properties support its broadspectrum benefits as a possible co- adjunctive supplement for COVID-19. However, at this stage we do not know whether nASX has any therapeutic value in the treatment or prevention of novel SARS-CoV-2 


\subsection{Food and drug safety of natural astaxanthin for human consumption}

H. pluvialis sourced natural ASX is reported as safe for human consumption, orally bioavailable, and a natural bio-active compound notified generally recognized as safe (GRAS) and approved by the United States Food and Drug Administration (USFDA) for human consumptions in dosages up to $24 \mathrm{mg}$ per day $(41,56)$. From the therapeutic point of view, nASX has been shown tremendous benefits against multiple disease without any reported side effects. Table 3 lists a few clinical reports involving nASX in human subjects.

\section{Conclusion}

COVID-19 caused by the infection of SARS-CoV-2 manifested by fever and pneumonia, leading to hyperinflammatory ARDS at severity is associated with SIRS or CRS, which suggest early prognosis and containment of disease progression. This necessitude a definitive anti-viral treatment along with a timely supportive anti-CRS or anti-inflammatory therapy to reduce the fatality of severe COVID-19 patients. While, several potential drugs for repurposing are undergoing clinical trials worldwide, a definitive COVID19 treatment is lacking altogether at present. Natural astaxanthin is a potent anti-inflammatory and antioxidant molecule with proven therapeutic and multiple health benefits anticipated to be useful as a coadjunctive supplement for COVID-19 patients. Neither astaxanthin has any role as antiviral nor any published evidence is available on its study against COVID-19. However, enough published evidences support its potential application to treat CRS and associated diseases. There is rationale to be anticipated its possible use as a potent co- adjunctive supplement for COVID-19, which requires further clinical evidences.

\section{Declarations}

\section{Declaration competing interest}

All authors declare no competing interests.

\section{Funding}

The authors did not receive any funding for the study

\section{Acknowledgement}

We thank IP team for prompt action to release the manuscript. 


\section{Ethical approval statement}

No ethical approval was obtained as all relevant information were retrieved from publicly available literature through multiple databases and no primary data was collected or generated during the review process.

\section{References}

1. Novel Coronavirus (2019-nCoV) situation reports - World Health Organization (WHO) https://www.who.int/

2. Liu T, Zhang J, Yang Y, Ma Hong, Li Z, Zhang J, Cheng J, Zhang X, et al. The potential role of IL-6 in monitoring severe case of coronavirus disease 2019. medRxiv preprint doi: https://doi.org/10.1101/2020.03.01.20029769

3. Huang C, Wang Y, Li X, et al. Clinical features of patients infected with 2019 novel coronavirus in Wuhan, China. Lancet 2020. https://doi.org/10.1016/S0140- 6736(20)30183-5.

4. Wong CK, Lam CW, Wu AK, Ip WK, Lee NL, Chan IH, Lit LC, Hui DS, Chan MH, Chung SS, Sung JJ. Plasma inflammatory cytokines and chemokines in severe acute respiratory syndrome. Clin Exp Immunol 2004; 136:95-103.

5. Fu Y, Cheng Y and Wu Understanding SARS-CoV-2 - Mediated inflammatory responses; From mechanism to potential therapeutic tools. Virologica Sinica 2020. https://doi.org/10.1007/s12250020-00207-4

6. Moore JB and June CH. Cytokine release syndrome in sever COVID-19. Science 2020. 1126/science.abb8925

7. Perlman S and Dandekar AA. Immunopathogenesis of coronavirus infections: implications for SARS. Nature Reviews, Immunology, 2005. doi:10.1038/nri1732

8. Fassett RG and Coobes JS. Astaxanthin: A Potential Therapeutic Agent in Cardiovascular Disease. Mar Drugs, 2011, 9, 447-465. doi:10.3390/md9030447

9. Miyachi M, Matsuno T, Asano K and Mataga I. Anti-inflammatory effects of astaxanthin in the human gingival keratinocyte line NDUSD1. 2015. doi: 10.3164/jcbn.14_109

10. Hussein G, Sankawa U, Goto H, Matsumoto K, and Watanabe H. Astaxanthin, a carotenoid with potential in human health and nutrition. J Nat Prod. 2006, 69(3):443-449.

11. Dhinaut J, Balourdet A, Teixeira M, Chogne M, Moret $Y$. A dietary carotenoid reduces immunopathology and enhances longevity through an immune depressive effect in an insect model, Scientific Rep. 2017. DOI:10.1038/s41598- 017-12769-7

12. Cai X, Chen Y, Xiaona Xie2, Yao D, et al. Astaxanthin prevents against lipopolysaccharide-induced acute lung injury and sepsis via inhibiting activation of MAPK/NF-KB. Am J Transl Res.

2019;11(3):1884-1894. PMID: $\underline{30972212}$ 
13. Zhang ZW, Xu XC, Liu T, Yuan S. Mitochondrion-Permeable Antioxidants to Treat ROS-Burst-Mediated Acute Diseases, 2016. http://dx.doi.org/10.1155/2016/6859523

14. Park JS, Chyun JH, Kim YK, Line LL, \& Chew BP. Astaxanthin decreased oxidative stress and inflammation and enhanced immune response in humans. Nutrition \& metabolism 2010, 7, 18. https://doi.org/10.1186/1743-7075-7-18

15. Davinelli S, Nielsen ME, \& Scapagnini G. Astaxanthin in Skin Health, Repair, and Disease: A Comprehensive Review. Nutrients 2018, 10(4), 522. https://doi.org/10.3390/nu10040522

16. Arksey H, O’Malley L. Scoping studies: towards a methodological International Journal of Social Research Methodology 2005, 8:19-32.

17. Moher D, et al. Preferred reporting items for systematic reviews and meta- analyses: the PRISMA statement. BMJ 2009, 339:332-336.

18. Li X, Geng B, Peng Y, Meng L, Lu S. Molecular immune pathogenesis and diagnosis of COVID-19. Journal of Pharmaceutical Analysis. 2020. https://doi.org/10.1016/j.jpha.2020.03.001

19. Wang D, Hu B, Hu C, Zhu F, Liu X, Zhang J, Wang B, et al. Clinical characteristics of 138 hospitalized patients with 2019 novel coronavirus-infected pneumonia in Wuhan, China. JAMA, 2020. doi:10.1001/jama.2020.1585.

20. Xu Z, Shi L, Wang Y, Zhang J, Huang L, Zhang C, et al. Pathological findings of COVID-19 associated with acute respiratory distress syndrome. The Lancet Respiratory medicine. 2020. https://doi.org/10.1016/S2213-2600(20)30076-X

21. Guan WJ, Ni ZY, Hu Y, Liang WH, Ou CQ, He JX, et al. Clinical Characteristics of Coronavirus Disease 2019 in China. The New England journal of medicine. 2020.

https://doi.org/10.1056/NEJMoa2002032.

22. Shi Y, Wang Y, Shao C, Huang J, Gan J, Huang X, Bucci E, et al. COVID-19 infection: the perspectives on immune responses. Cell Death \& Differ. 2020. https://doi.org/10.1038/s41418-020-0530-3

23. Liu Y, Yang Y, Zhang C, Huang F, Wang F, Yuan J, Wang Z, et al. Clinical and biochemical indexes from 2019-nCoV infected patients linked to viral loads and lung injury. Sci. China Life Sci. 2020; 63: 364-374.

24. Bonow RO, Fonarow GC, O'Gara PT, Yancy CW. Association of Coronavirus Disease 2019 (COVID-19) With Myocardial Injury and Mortality. JAMA Cardiol. 2020. doi:10.1001/jamacardio.2020.1105

25. Chen N, Zhou M, Dong X, et al. Epidemiological and clinical characteristics of 99 cases of 2019 novel coronavirus pneumonia in Wuhan, China: a descriptive study. Lancet 2020; 395: 507-13.

26. Chan KW, Wong VT and Tang CW. COVID-19: An update on the epidemiological, clinical, preventive and therapeutic evidence and guidelines of integrative Chinese - Western medicine for the management of 2019 novel coronavirus disease. DOI: 1142/S0192415X20500378

27. Zhou F, Yu T, Du R, Fan G, et al. Clinical course and risk factors for mortality of adult inpatients with COVID-19 in Wuhan, China: a retrospective cohort study. https://doi.org/10.1016/S01406736(20)30566-3 
28. MacLaren G, Fisher D, Brodie D. Preparing for the most critically III patients with COVID-19: the potential role of extracorporeal membrane oxygenation. JAMA. 2020.

https://doi.org/10.1001/jama.2020.2342

29. Zhang K. Is traditional Chinese medicine useful in the treatment of COVID-19? Am J Emerg Med. 2020. doi: 1016/j.ajem.2020.03.046

30. Lu H. Drug treatment options for the 2019-new coronavirus (2019-nCoV). Biosci. Trends 2020. https://doi.org/10.5582/bst.2020.01020.

31. Arabi YM, Asiri AY, Assiri AM, Aziz HA. Treatment of Middle East respiratory syndrome with a combination of lopinavir/ ritonavir and interferon- $\beta 1 \mathrm{~b}$ (MIRACLE trial): statistical analysis plan for a recursive two stage group sequential randomized controlled trial. https://doi.org/10.1186/s13063019-3846-x

32. Gao J, Tian Z, Yang X. Breakthrough: Chloroquine phosphate has shown apparent efficacy in treatment of COVID-19 associated pneumonia in clinical studies. Trends. 2020;14, 72-73. DOI:10.5582/bst.2020.01047

33. Wang M, Cao R, Zhang L, Yang X, Liu J, Xu M, Shi Z, Hu Z, Zhong W, Xiao Remdesivir and chloroquine effectively inhibit the recently emerged novel coronavirus (2019-nCoV) in vitro. Cell Res. 2020

34. Zheng C, Wang J, Guo H, Lu Z, Ma Y, Zhu Y, Xia D, et al. Risk adapted strategy for COVID-19 patients. Int J Infect Dis. 2020. https://doi.org/10.1016/j.ijid.2020.03.047

35. Zhang W, Zhao Y, Zhang F, Wang Q, Li T, Liu Z, et al. The use of anti-inflammatory drug in the treatment of people with sever coronavirus disease 2019 (COVID-19): The perspectives of clinical immunologists from China, Clinical Immun. 2020. https:/ doi.org/10.1016/j.clim.2020.108393

36. Zhang R, Wang X, Ni L, et al. COVID-19: Melatonin as a potential adjuvant treatment, Life Sciences 2018. https://doi.org/10.1016/j.lfs.2020.117583

37. Pashkow FJ, Watumull DG, Campbell CL. Astaxanthin: A novel potential treatment for oxidative stress and inflammation in cardiovascular disease. Am. J. Cardiol. 2008, 101, 58-68.

38. Sztretye M, Dienes B, Gönczi M, et al. Astaxanthin: A Potential Mitochondrial-Targeted Antioxidant Treatment in Diseases and with Aging. Oxidative Medicine and Cellular Longevity 2019. https://doi.org/10.1155/2019/3849692

39. Barros MP, Poppe SC, Bondan EF. Neuroprotective properties of the marine carotenoid astaxanthin and omega-3 fatty acids, and perspectives for the natural combination of both in krill oil. Nutrients 2014(6):1293-1317. doi:10.3390/nu6031293

40. Chew BP, Park JS. The immune system and disease. In Carotenoids: Nutrition and Health; Birkhauser Press: Basel, Switzerland, 2009; Volume 5, pp. 363-382.

41. Capelli B, Talbott S, and Ding L. Astaxanthin sources: suitability for human health and nutrition. Functional Foods in Health and Disease 2019;9(6):p

42. Rosenbaugh EG, Savalia KK,Manickam DS and Zimmerman MC. Antioxidant- based therapies for angiotensin II-associated cardiovascular diseases, Am J Physiol Regul Integr Comp Physiol. 2013. 
doi:10.1152/ajpregu.00395.2012

43. Cui $\mathrm{H}$, Kong $\mathrm{Y}$, Zhang H. Oxidative stress, mitochondrial dysfunction, and aging. Journal of Signal Transduction. 2012, Article ID

44. Hoffman R, Sultan LD, Saada A, et al. Astaxanthin extends lifespan via altered biogenesis of the mitochondrial respiratory chain complex III. https://doi.org/10.1101/698001

45. Wu H, Niu H, Shao A, Wu C, Dixon BJ, Zhang J, Yang S, Wang Y. Astaxanthin as a Potential Neuroprotective Agent for Neurological Diseases. Mar drugs 2015 (13): 5750-5766.

46. Kouhpayeh S, Shariati L, Boshtam M, Rahimmanesh I, Mirian M, et al. The role of COVID-19; NAD+ depletion addresses all question in this infection. 2020 doi:10.20944/preprints202003.0346.v1

47. Montezano AC, Touyz RM. Reactive oxygen species and endothelial function- role of nitric oxide synthase uncoupling and Nox family nicotinamide adenine dinucleotide phosphate oxidases. Basic Clin Pharmacol Toxicol110: 87-94, 2012.

48. Guo F, Chen XL, Wang F, Liang X, Sun YX, Wang YJ. Role of angiotensin II type 1 receptor in angiotensin II-induced cytokine production in macrophages. J Interferon Cytokine Res 31: 351-361,

49. Lee MY, Griendling KK. Redox signaling, vascular function, and Antioxid Redox Signa/ 10: 10451059, 2008.

50. Tan W and Aboulhosn J. The cardiovascular burden of coronavirus disease 2019 (COVID-19) with a focus on congenital heart disease. Int J Card 2020. https://doi.org/10.1016/j.ijcard.2020.03.063

51. Song $X$, Wang B, al. Astaxanthin inhibits apoptosis in alveolar epithelial cells type II in vivo and in vitro through the ROS-dependent mitochondrial signaling pathway, J. Cell. Mol. Med. 2014;18(11):2198-2212. doi: 10.1111/jcmm.12347

52. Cheresh P, Kim SJ, Tulasiram S, et al. Oxidative stress and pulmonary fibrosis. Biochim Biophys Acta. 2013; 1832: 1028-40.

53. Nishida Y, Yamashita E, Miki W. Quenching activities of common hydrophilic and lipophilic antioxidants against singlet oxygen using chemiluminescence detection system, Carotenoid Science 2007;11(6):16-20.

54. Coral-Hinostroza GN, Ytrestoyl T, Ruyter B, Bjerkeng B. Plasma appearance of unesterified astaxanthin geometrical $E / Z$ and optical $R / S$ isomers in men given single doses of a mixture of optical 3 and 3'R/S isomers of astaxanthin fatty acyl diesters. Comp Biochem Physiol C Toxicol Pharmacol. 2004;139:99-110.

55. Zhang X, Yue Lu Y, Wu Q, et al. Astaxanthin mitigates subarachnoid hemorrhage injury primarily by increasing sirtuin 1 and inhibiting the Toll-like receptor 4 signaling pathway. 2018. doi: 10.1096/fj.201800642RR

56. Kidd P. Astaxanthin, cell membrane nutrient with diverse clinical benefits and anti-aging potential. Altern. Med. Rev. 2011; 16:355-364.

57. Lee SJ, Bai SK, Lee KS, et al. Astaxanthin inhibits nitric oxide production and inflammatory gene expression by suppressing I(kappa)B kinase dependent NF- kappaB activation. Mol Cells 2003; 16 : 
97-105.

58. Roopashree MR, Gondhalekar RV, Shashikanth MC, George J, et al. Pathogenesis of oral lichen planus - J Oral Pathol Med 2010; 39: 729- 734.

59. Femiano F and Scully C. Functions of cytokines in relation oral lichen planus- hepatitis C. Med Oral Patol Oral Cir Bucal 2005; 10:E40-E44.

60. Giuliani M, Lajolo C, Saroria A, et al. Oral lichenoid lesions in HIV-HCV- coinfected subjects during antiviral therapy: 2 cases and review of literature. Am J Dematopathol 2008; 30: 466-471.

61. Yang $\mathrm{C}$, Hassan $\mathrm{Yl}$, Liu R, Zhang $\mathrm{H}$, Chen $\mathrm{Y}$, et al. Anti-inflammatory effects of different astaxanthin isomers and the roles of lipid transporters in the cellular transport of astaxanthin isomers in Caco-2 Cell monolayers. J Agric Food Chem. 2019; 67(22): 6222-6231. https://doi.org/10.1021/acs.jafc.9b02102

62. Chan KC, Pen PJ, Yin MC. Anticoagulatory and anti-inflammatory effects of astaxanthin in diabetic rats. J Food Sci 2012; 77: H76-H80.

63. Matthay MA, Aldrich JM, Gotts JE. Treatment of severe acute respiratory syndrome from COVID-19, The Lancet. 2020. https://doi.org/10.1016/S2213- 2600(20)30127-2

64. Abdulamir AS and Hafidh RR. The possible immunological pathway for the variable immunopathogenesis of COVID-19 infections among healthy adults, elderly and children. Electron J Gen Med. 2020;17(4):em202. https://doi.org/10.29333/ejgm/7850

65. Alhazzani W, Møller MH, ArabiYM, Loeb M, al. Surviving Sepsis Campaign: guidelines on the management of critically ill adults with Coronavirus Disease 2019 (COVID-19). Intensive Care Med. 2020. https://doi.org/10.1007/s00134- 020-06022-5

66. Williams AE, Chambers RC, The mercurial nature of neutrophils: still an enigma in ARDS? Am. J. Physiol. Lung Cell Mol. Physiol. 2014. https://doi.org/10.1152/ajplung.00311.2013.

67. Chaplin DD. Overview of the immune response. J Allergy Clin Immunol. 2010. doi:10.1016/j.jaci.2009.12.980.

68. Park JS, Kim HW, Mathison BD et al. Astaxanthin uptake in domestic dogs and cats. Nutrition \& Metabolism 2010;7(1):52.

69. Chew BP, Wong MW, Park JS, Wong TS. Dietary b-carotene and astaxanthin but not canthaxanthin stimulate splenocyte function in mice. Anticancer Re 1999, 19:5223-5227.

70. Lin et al. Astaxanthin, a Carotenoid, Stimulates Immune Responses by Enhancing IFN- and IL-2 Secretion in Primary Cultured Lymphocytes in Vitro and ex Vivo. 2016. doi:10.3390/ijms17010044

71. Diao W, Chen W, Cao W, Yuan H, Ji H, Wang T, al. Astaxanthin protects against renal fibrosis through inhibiting myofibroblast activation and promoting CD8+ T cell recruitment, 2019. DOI: 10.1016/j.bbagen.2019.05.020

72. Jyonouchi H, Zhang L, Gross M, Tomita Y. Immunomodulating actions of carotenoids: enhancement of in vivo and in vitro antibody production to T- dependent antigens. Nutr Cancer 1994, 21:47-58. 
73. Mehta P, McAuley MB, Sanchez E, et al. COVID-19: consider cytokine storm syndromes and immunosuppression. The Lancet 2020. https://doi.org/10.1016/S0140-6736(20)30630-9

74. Kowshik J, Baba AB, Giri H, Reddy GD, Dixit M, et al. Astaxanthin inhibits JAK/STAT-3 signaling to abrogate cell proliferation, invasion and angiogenesis in a Hamster model of oral cancer. PLoSONE 9(10): e109114. doi:10.1371/journal.pone.0109114

75. Fakhri S, Aneva IY, Faizal MH, al. The Neuroprotective Effects of Astaxanthin: Therapeutic Targets and Clinical Perspective. Molecules 2019, 24, 2640; doi:10.3390/molecules24142640

76. Hussein G, Nakamura M, Zhao Q, Iguchi T, Goto H, Sankawa U, Watanabe H. Antihypertensive and neuroprotective effects of astaxanthin in experimental animals. Biol Pharm Bull. 2005 Jan;28(1):4752.

77. Wu Y, Xu X, Chen Z, Duan J, Hashimoto K, et al. Nervous system involvement after infection with COVID-19 and other coronaviruses. Brain, Behavior, and Immunity 2020. doi: https://doi.org/10.1016/j.bbi.2020.03.031

78. Mao L., Wang M.D., Chen S.H., He Q.W., Chang J., Hong C.D., et al., 2020. Neurological manifestations of hospitalized patients with COVID-19 in Wuhan, China: a retrospective case series study. MedRxiv 02.22.20026500

79. Xiang P, Xu XM, Gao LL, Wang HZ, Xiong HF, Li RH, et al. First Case of 2019 Novel Coronavirus Disease with Encephalitis. ChinaXiv 2020. T202003.00015.

80. Zhang XS, Zhang X, Zhou ML, Zhou XM, Li N, Li W, Cong ZX, et al. Amelioration of oxidative stress and protection against early brain injury by astaxanthin after experimental subarachnoid hemorrhage. J. Neurosurg. 2014, 121, 42-54.

81. Fakhri S, Dargahi L, Abbaszadeh F, Jorjani M. Astaxanthin attenuates neuroinflammation contributed to the neuropathic pain and motor dysfunction following compression spinal cord injury. Brain Res. Bull. 2018,143, 217-224

82. Zhang XS, Zhang X, Zhang QR, Wu Q, Li W, Jiang TW, et al. Astaxanthin reduces matrix metalloproteinase- 9 expression and activity in the brain after experimental subarachnoid hemorrhage in rats. Brain Res. 2015, 1624, 113-124.

83. Gao D, Yao S, Sun Y, Zheng Di, Zhang Q, Li W. Astaxanthin attenuate iohexol- induced human proximal renal tubular epithelial cells injury via the ROS/NLRP3 inflammasome signal pathway. SDRP Journal of Food Science \& Technology 2019;4(3)

84. Ding T, Wang S, Zhang X, Zai W, Fan J, Chen W, Bian Q, Luan J, Shen Y, Zhang Y, Ju D and Mei X. Kidney protection effects of dihy-droquercetin on diabetic nephropathy through suppressing ROS and NLRP3 inflammasome. Phytomedicine 2018; 41: 45-53. PMid:29519318

85. Xu W, Wang M, Cui G, Jiao D, Yao B, Xu K, et al. Astaxanthin protects OTA-induced lung injury in mice through Nrf2/NF-kB pathway, Toxins 2019, 11(9), 540; https://doi.org/10.3390/toxins11090540

86. Kubo H, Asai K, Kojima K, Kyomoto Y, et al. Astaxanthin suppresses cigarette smoke- induced Emphysema through Nrf2 activation in mice. Marine Drugs 2019. doi:10.3390/md17120673 
87. Spiller G, Dewell A, Chaves S, Rakidzich Z. Effect of daily use of natural Astaxanthin on C-reactive protein. Unpublished study, 2006, cited in "The World's Best Kept Health Secret: Natural Astaxanthin" Capelli B and Cysewski G. (2014).

88. Baralic I, Andjelkovic M, Djordjevic B, Dikic N, Radivojevic N, Suzin-Zivkovic V, Radojevic-Skodric S, Pejic S. Effect of Astaxanthin supplementation on Salivary IgA, oxidative stress, inflammation in young soccer players. Evidence Based Complementary and Alternative Medicine 2015; 2015:783761.

89. Iwamoto T, Hosoda K, Hirano R, Kurata H, Matsumoto A, Miki W, Kamiyama M, Itakura H, Yamamoto S, Kondo K. Inhibition of low-density lipoprotein oxidation by astaxanthin. J Atheroscler Thromb. 2000, 7, 216-222.

90. Mercke Odeberg J, Lignell A, Pettersson A, Hoglund P. Oral bioavailability of the antioxidant astaxanthin in humans is enhanced by incorporation of lipid-based formulations. Eur. J. Pharm. Sci. 2003, 19, 299-304.

91. Spiller GA, Dewell A. Safety of an astaxanthin-rich Haematococcus pluvialis algal extract: A randomized clinical trial. Med. Food 2003, 6, 51-56.

92. Miyawaki H, Takahashi J, Tsukahara H, Takehara I. Effects of astaxanthin on human blood rheology. Clin. Biochem. Nutr. 2008, 43,69-74.

93. Deng ZY, Shan WG, Wang SF, Hu MH, Chen Y. Effect of astaxanthin on blood coagulation, fibrinolysis and platelet aggregation in hyperlipidemic rats. Pharmaceutical Biology, 2017; 55(1):663-672. http://dx.doi.org/10.1080/13880209.2016.1261905

\section{Tables}

Due to technical limitations, the tables are only available as a download in the supplemental files section.

\section{Figures}

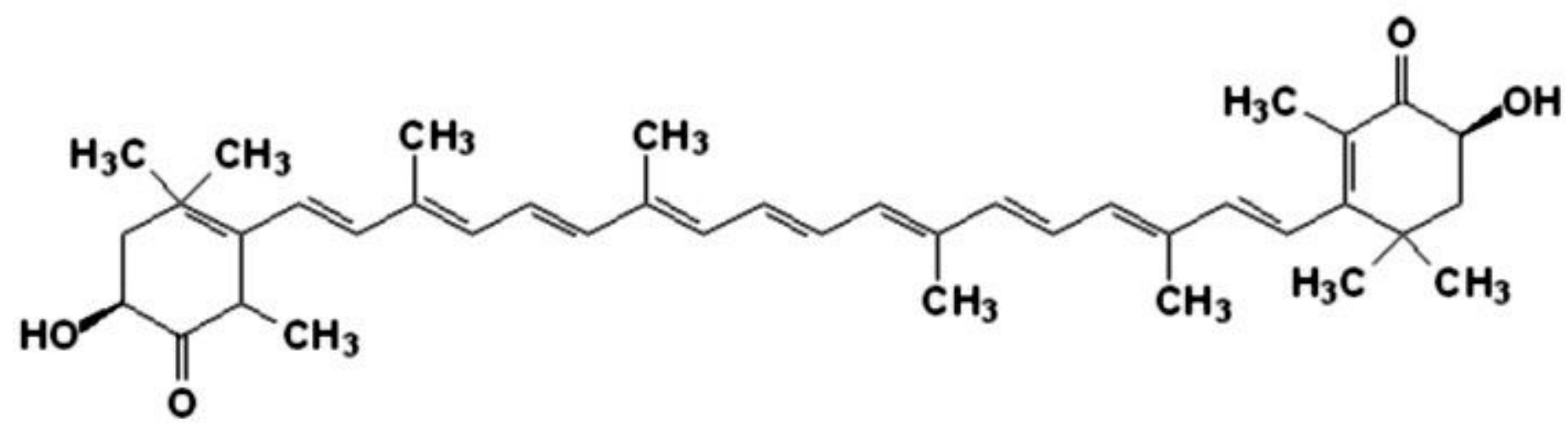

\section{Figure 1}

Chemical structure of astaxanthin 


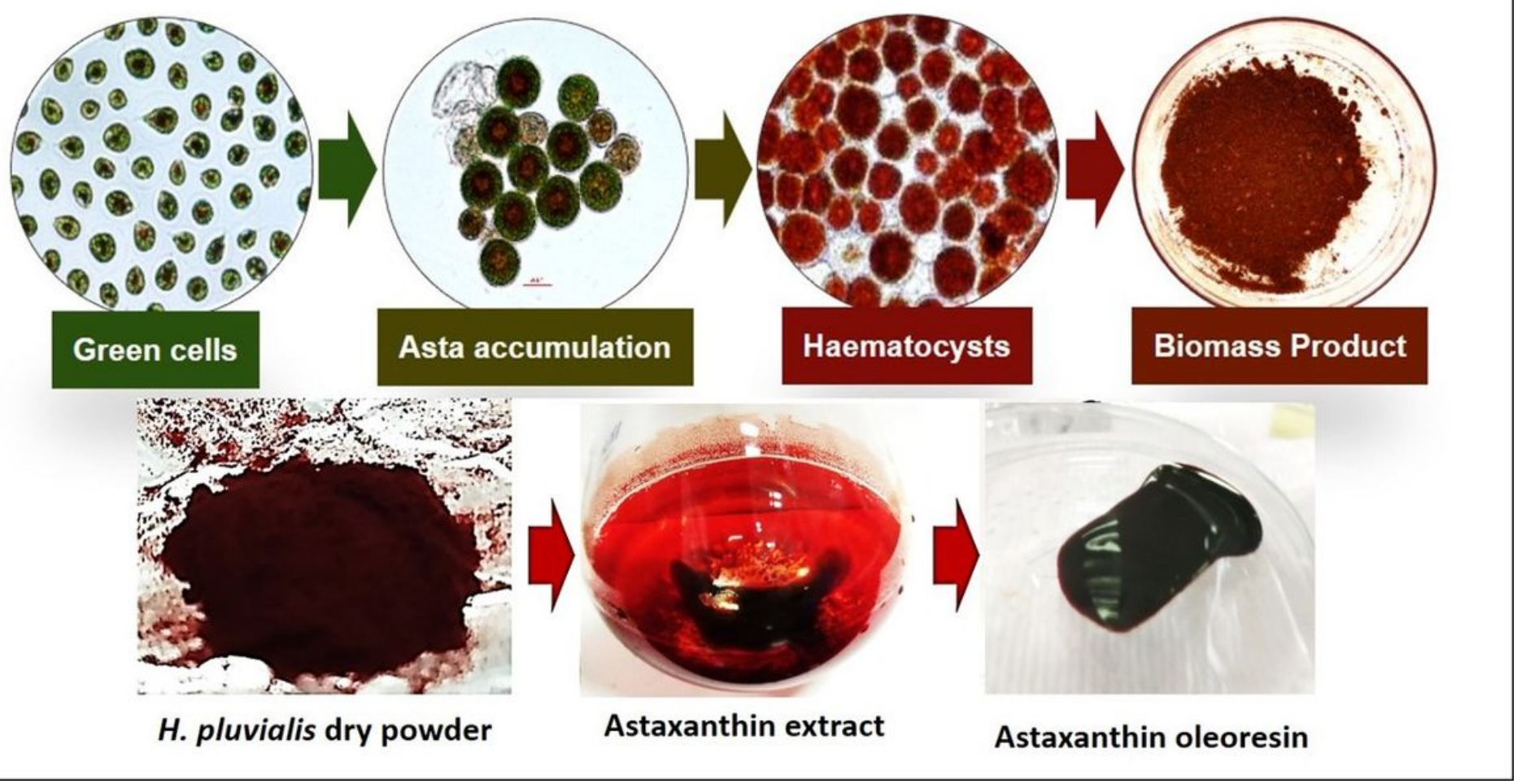

Figure 2

An outline showing the various stages of production of astaxanthin from microalgae H.pluvialis. 


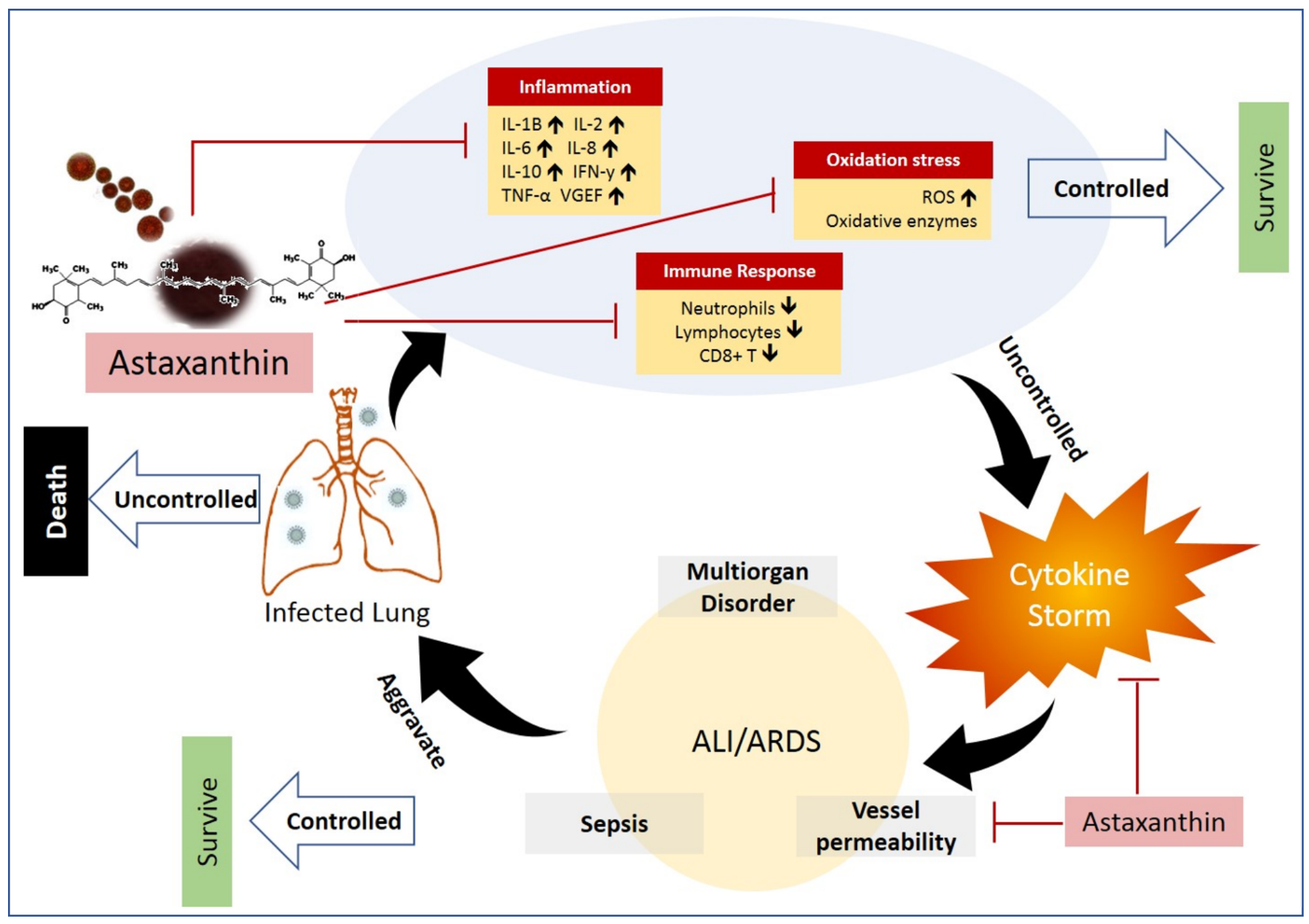

Figure 3

Pathogenesis of COVID-19 and potential use of natural astaxanthin. We presumed that lung infected by SARS-CoV-2 elevated oxidation stress, elevated ROS mediated inflammation and a suppressed immune response proceed unabated resulting violent cytokine storm. ARDS/ALI may ensue, accompanied by series of complications, which vary according to the disease severity. Astaxanthin may play the vital role in regulation of the oxidative stress induced by ROS, regulation of the immune response, and downregulation of pro-inflammatory components, resulting in alleviation of cytokine storm. Astaxanthin may also provide support for patients with ALI/ARDS and related complications with its antiinflammatory properties.

\section{Supplementary Files}

This is a list of supplementary files associated with this preprint. Click to download.

- S2PRISMAScRFillableChecklist5May2020.docx

- Tables.pdf 
- S1Classificationretrieveddata.jpg

- S2PRISMAFlowChartshowingStudySelectionProcess.jpg 